\title{
A Systems Chemical Biology Study of Malate Synthase and Isocitrate Lyase Inhibition in Mycobacterium tuberculosis During Active and NRP Growth
}

\author{
Elebeoba E. May ${ }^{1, *}$, Andrei Leitão ${ }^{2}$, Alexander Tropsha $^{3}$, and Tudor I. Oprea ${ }^{4}$ \\ ${ }^{1}$ Biomedical Engineering Department, University of Houston, 3605 Cullen Blvd., Houston, TX \\ 77204 USA \\ ${ }^{2}$ Medicinal Chemistry Group -(NEQUIMED), Institute of Chemistry of Sao Carlos, University of \\ Sao Paulo, Sao Carlos, SP, Brazil \\ ${ }^{3}$ Laboratory for Molecular Modeling, Division of Medicinal Chemistry and Natural Products, \\ Eshelman School of Pharmacy, University of North Carolina at Chapel Hill, Chapel Hill, NC 27599 \\ ${ }^{4}$ Department of Biochemistry and Molecular Biology, University of New Mexico School of \\ Medicine, MSC11 6145, Albuquerque NM 87131, USA
}

\section{Abstract}

The ability of Mycobacterium tuberculosis (Mtb) to survive in low oxygen environments enables the bacterium to persist in a latent state within host tissues. In vitro studies of Mtb growth have identified changes in isocitrate lyase (ICL) and malate synthase (MS) that enable bacterial persistent under low oxygen and other environmentally limiting conditions. Systems chemical biology (SCB) enables us to evaluate the effects of small molecule inhibitors not only on the reaction catalyzed by malate synthase and isocitrate lyase, but the effect on the complete tricarboxylic acid cycle (TCA) by taking into account complex network relationships within that system.

To study the kinetic consequences of inhibition on persistent bacilli, we implement a systemschemical biology (SCB) platform and perform a chemistry-centric analysis of key metabolic pathways believed to impact Mtb latency. We explore consequences of disrupting the function of malate synthase (MS) and isocitrate lyase (ICL) during aerobic and hypoxic non-replicating persistence (NRP) growth by using the SCB method to identify small molecules that inhibit the function of MS and ICL, and simulating the metabolic consequence of the disruption.

Results indicate variations in target and non-target reaction steps, clear differences in the normal and low oxygen models, as well as dosage dependent response. Simulation results from singular and combined enzyme inhibition strategies suggest ICL may be the more effective target for

(C) 2013 Elsevier Ltd. All rights reserved.

*Corresponding author: eemay@ central.uh.edu.

Publisher's Disclaimer: This is a PDF file of an unedited manuscript that has been accepted for publication. As a service to our customers we are providing this early version of the manuscript. The manuscript will undergo copyediting, typesetting, and review of the resulting proof before it is published in its final citable form. Please note that during the production process errors may be discovered which could affect the content, and all legal disclaimers that apply to the journal pertain. 
chemotherapeutic treatment against Mtb growing in a microenvironment where oxygen is slowly depleted, which may favor persistence.

\section{Keywords}

Biological networks; cheminformatics; biochemical network simulations; systems biology; chemical biology; Mycobacterium tuberculosis

\section{Introduction}

Mycobacterium tuberculosis (Mtb), the causative agent of tuberculosis (TB), is able to persist in host tissues in a non-replicating persistence (NRP) or latent state, with 2 billion people estimated to serve as a reservoir for the bacterium [Jasmer et al., 2002]. This presents a challenge in the treatment of TB and latent TB specifically, which has a re-activation rate of ten percent for individuals with normal immune systems, higher for those with compromised immune systems. Previous and current studies of Mtb attempt to identify and analyze mechanisms that enable the bacterium to survive within a presumably low oxygen, low nutrient, and acidic microenvironment created as a result of host-response to infection [Cosma, et al. 2003; Deb, et al. 2009; Schnappinger, et al. 2006]. Researchers have used theoretical models and quantitative analysis of Mtb metabolism and latency-associated biochemical pathways to integrate empirical data into models that can provide additional insight on how various mechanisms interact to enable the bacilli to survive under harsh physiological conditions [Belta, et al. 2003; Beste, et al. 2007; Singh and Ghosh, 2006]. Computational models that analyze the impact of enzyme inhibition on Mtb fatty acid and iron metabolism pathways, and consequentially on Mtb growth, have been developed using dynamic flux balance analysis methods to capture the metabolic consequences of inhibition [Fang et al., 2009; Fang et al., 2011]. Improving and expanding the level of chemistry awareness in these models through the inclusion of cheminformatics and pharmacokinetics data in theoretical models and analysis platforms will allow scientists to explore possible means for disrupting metabolic mechanisms that enable Mtb persistence. Systems chemical biology (SCB), the integration of systems biology and chemical biology [Oprea, et al 2007], and computational systems biology, recently described in [Oprea, et al. 2011], provide tools for developing SCB platforms for analysis of biological systems. In this work we use the SCB methodology to study the interruption of malate synthase and isocitrate lyase in Mtb during aerated growth and low oxygen growth resulting in non-replicating persistence (Figure 1).

These two enzymes are part of Mtb's glyoxylate bypass, a particularly attractive therapeutic target due to the importance of this pathway to Mtb survival during a persistent infection and the absence of this pathway in mammalian cells [Smith, et al., 2003]. Combining our understanding of metabolic pathways that contribute to Mtb survival with information on how small molecules and chemotherapeutic agents disrupt these pathways will aid in the development of more effective methods to counter and reduce TB associated fatalities. 


\subsection{Metabolism and Mtb Persistence}

Studies of Mtb metabolism indicate that the glyoxylate bypass, which consists of two reaction steps catalyzed by isocitrate lyase (ICL, gene $i c l$ ) and malate synthase (MS, gene $g l c B)$, is a key metabolic pathway that may enable Mtb to adapt to low nutrient conditions and low oxygen or hypoxic conditions [Smith, et al. 2003; Wayne and Hayes, 1996; Wayne and Sohaskey, 2001]. During growth on glucose, the tricarboxylic acid cycle (TCA) cycle uses carbon moieties produced through glycolysis to generate reducing agents (NADH, FADH2) that are used to produce cellular energy in the form of ATP by way of the electron transport chain (ETC) and oxidative phosphorylation [Nelson and Cox, 2005]. The generation of reducing agents for oxidative phosphorylation through the TCA cycle requires oxidizing agents (NAD, FAD), which are proportionately regenerated during oxidative phosphorylation and are important metabolic cofactors. In addition, oxaloacetate and 3ketoglutarate generated in the TCA cycle can feed into the amino acid biosynthesis pathway. During low nutrient and low oxygen conditions Mtb presumably needs alternative mechanisms for generating sufficient cellular energy and regenerating redox and cofactor molecules.

The Wayne in vitro model of non-replicating persistence (NRP) suggests that up regulation of ICL may replenish oxidative cofactors through alternative NAD generation pathways activated in the oxygen limited bacilli [Wayne and Lin, 1982; Wayne and Hayes, 1996; Wayne and Sohaskey, 2001]. Wayne and colleagues observed that during hypoxic growth conditions isocitrate lyase (ICL) increased five-fold, however a comparable increase in the second enzyme in the pathway, malate synthase (MS) was not observed. Thus they hypothesized that the increase in ICL and subsequent increase in glyoxylate may serve to replenish NAD by way of the glyoxylate-to-glycine (GtG) shunt. The observed ten-fold increase in glycine dehydrogenase (GDH), the key enzyme in the GtG shunt, during slow stirred NRP growth further substantiated their argument. An alternative explanation suggests that the glyoxylate bypass may be used to generate malate to move into gluconeogenesis, which would presumably replenish NAD [Smith, et al. 2003].

While increase in glyoxylate may partially serve to replenish NAD, low nutrient conditions would reduce the carbon-containing molecules entering the TCA cycle through the glycolysis pathway. Glycolysis-produced pyruvate is converted to acetyl-CoA, and acetylCoA enters the TCA cycle. Mtb's mycolic acid cell wall may reduce the reliance on glycolysis in favor of beta-oxidation for production of acetyl-CoA via fatty acid metabolism. The glyoxylate pathway combined with malate synthase conversion of glyoxylate to malate allows Mtb to bypass two energy-requiring steps of the standard TCA cycle while producing the necessary intermediates to maintain the cycle [Smith, et al. 2003]. In addition the bypass conserves carbon while producing necessary biosynthetic precursors such as oxaloacetate, an important substrate in the synthesis of amino acids. Therefore both glyoxylate and malate, and by extension isocitrate lyase and malate synthase, are important for sufficient regeneration of oxidizing cofactors for energy production and conservation of carbon moieties during Mtb growth under low oxygen and low nutrient conditions. We explore consequences of disrupting the function of Mtb's malate synthase and isocitrate lyase enzymes during aerobic active growth and anaerobic NRP growth using the SCB method to 
study how small molecules can inhibit the function of these two enzymes. We simulate the metabolic consequences of the disruption and evaluate how addition of small molecules impacts metabolites required for bacterial survival.

\section{Materials and Methods}

As described by [Oprea, et al. 2011] the goal of computational SCB is to develop platform tools that integrate cheminformatics and systems biology data acquisition, processing, and integration into a theoretical framework for scientific exploration. In this study we apply two key computational platforms used in SCB: 1) modeling and simulation of Mtb's TCA cycle and glyoxylate bypass network; 2) use of cheminformatics to perform virtual screening of inhibitory molecules that interact with enzymes.

\subsection{Simulating Mtb during active growth and non-replicating persistence}

We develop a theoretical model of the TCA cycle, glyoxylate bypass, and the glyoxylate-toglycine shunt (depicted in Fig.1, reactions listed in Table 1) based on the Singh and Ghosh model, the Wayne NRP model, and data from Beste, et al.'s reconstructed metabolic network of Mtb [Singh and Ghosh, 2006; Wayne and Hayes, 1996; Beste, et al. 2007].

The model explicitly incorporates NAD/NADH and FAD/FADH2 substrates and uses updated kinetic parameters from the BRENDA Enzyme database and empirical data on Mtb growth under low oxygen conditions [Scheer, et al., 2011; Wayne and Hayes, 1996; Smith, et al. 2003]. We use traditional Michaelis-Menten assumptions [Nelson and Cox, 2005] to determine reaction rate equations. Parameters are based on values used in the Singh-Ghosh model, growth rates and conditions reported for the Wayne NRP model, or kinetic values specified in the BRENDA Enzyme Database. We use the relation $V_{M a x}=K_{c a t} \times E_{\text {total }}$, where the enzyme concentration used in the simulation is $E_{\text {total }} \times 10^{-2}$, making the reaction rate (e.g. for $\mathrm{S}=\mathrm{P}$ reaction): $V=\frac{V_{M a x} \times 10^{-2}[S]}{[S]+K_{M}}$. To capture empirically observed fold changes in the glyoxylate bypass enzymes (ICL, MS, GDH) due to growth under low oxygen conditions we multiply $V$ by the fold change value. We capture oxygen-mediated regeneration of NAD/FAD by approximating the net effect of oxidative phosphorylation using the following stoichiometric relation: $\mathrm{NADH}+1 / 2 \mathrm{O}_{2}=\mathrm{NAD} ; \mathrm{FADH}_{2}+1 / 2 \mathrm{O}_{2}=\mathrm{FAD}$. The relative amount of available oxygen, $\left[\mathrm{O}_{2}\right]$, is determined using an initial oxygen concentration of $8.0 \mathrm{e}-5 \mathrm{mM}$ and oxygen depletion data for Mtb grown under aerobic, slowstirred hypoxic, and vigorously agitated hypoxic conditions reported in the Wayne NRP models. The initial oxygen concentration value was calculated as twice the $\mathrm{K}_{\mathrm{M}}$ value of $\mathrm{O}_{2}$ for the Cytochrome C oxidase (EC 1.9.3.1) as reported in BRENDA for Helicobacter pylori.

We use a circuit based simulation platform, BioXyce, to implement the model [May and Schiek, 2009; May 2011]. At the cellular level, biochemical pathways are modeled as electrical circuits where signals are produced, propagated and consumed. BioXyce uses the following equivalents: chemical mass as charge, mass flux as electric current, concentration as voltage, stoichiometric conservation as Kirchhoff's voltage law, and mass conservation as Kirchhoff's current law. With BioXyce, we can focus on subsystems as described in this 
work as well as simulate large control networks consisting of entire cells, heterogeneous, and homogeneous cell populations.

\subsection{The Cheminformatics Workflow}

The second aspect of computational SCB used in this work is the use of an automatable cheminformatics pipeline to screen for small molecule inhibitors of the malate synthase enzymes and identification of critical parameters associated with the inhibitors. Figure 2 illustrates the workflow used to obtain information regarding the ligand-protein interactions of interest in this work:

First we perform searches in WOMBAT and SciFinder using names or Enzyme Commission (EC) numbers of the enzymes in order to retrieve small molecules with relevant biological activity. We consider different protonation states for the molecules. Most of the compounds have a carboxylic acid. Using the Omega software package (OpenEye Scientific Software, Santa Fe, NM) [Boström, J. et al 2003; Hawkins 2010, 2012] we generate three-dimensional conformations for the set of compounds. For enzymes in the glyoxylate cycle, we use the Protein Data Bank (PDB) as the source for 3D structures of malate synthase (2GQ3) and isocitrate lyase $(1 \mathrm{~F} 8 \mathrm{M})$. Mutations in the sequence of each protein are checked by aligning them in ClustalW with the reference amino acid sequences. Next we prepare the enzymes and perform docking analysis (Fig. 3) with FRED (version 2.1 (OpenEye Scientific Software, Santa Fe, NM) [McGann, M. et al 2003] generating a total of 500 poses and saving 20 alternative poses for each molecule after using the chemgauss 3 scoring function, keeping default values to the remaining parameters. Two docking conditions are tested: (1) after removal of the water molecules in the active pocket; (2) considering the water molecules in the pocket.

Before running any docking study using crystallographic water molecules, we analyzed the enzyme pockets with GRID v. 22 (Molecular Discovery Inc., London) to better understand the environment of the active pockets and to determine the most important crystallographic water molecules to keep during docking. This was accomplished by using a set of virtual probes in default mode (water, carbonyl oxygen, amide nitrogen, hydrophobic and Csp2) to compute the interaction energies between the probe and the amino acids from the enzyme at the lattices of a grid cage.

The docking results are used to determine how well a candidate ligand fits in the enzyme pocket and the plausibility of the putative inhibitor pose. This process is used to identify potential inhibitors of the enzyme of interest, which are then incorporated into the theoretical model of the system described in Section 2.1.

\section{Results}

Systems chemical biology (SCB) enables us to evaluate the effects of small molecule inhibitors on the reaction catalyzed by malate synthase and isocitrate lyase, as well as the indirect effect of inhibition on the complete TCA cycle by taking into account complex network relationships within that system. We first use the SCB platform to perform a chemistry-centric analysis of key metabolic pathways believed to impact Mtb latency. The 
resulting cheminformatics models can help identify potential inhibitors of this pathway based on available chemical, biological and structural information. Finally, we incorporate the inhibitors into our metabolic network model to enable comparison of normal and small molecule-inhibited Mtb growing under aerated and low oxygen conditions, conditions selected to replicate the Wayne aerated/NRP experimental system.

\subsection{Identification of small molecule inhibitors using SCB-related virtual screening studies}

To enable chemistry cognizance in the $M t b$ pathway simulations, we apply virtual screening as previously described to identify small bioactive molecules. We take advantage of the presence of 3D structures (from X-ray crystallography) for two of the enzymes in the $M t b$ glyoxylate shunt, namely malate synthase and isocitrate lyase. In this section we will illustrate the process using our analysis of the MS enzyme. The substrate binding site in each enzyme is evaluated using the GRID program. Malate synthase (PDB entry 2GQ3) has an active site that accommodates the substrate (glyoxylate) and the co-factor, acetyl-CoA, in order to release malate and CoA (Fig. 4).

This site, already subjected to investigation [Mdluli and Spigelman, 2006], features a relatively small number of hydrophobic interactions, which suggests that classical inhibitor design methods may prove unsuccessful. Besides that, we detected another cavity in the vicinity of the catalytic site, which may function as an allosteric site and is more hydrophobic. This potentially new allosteric site may be subject to the design of novel modulators in the future, especially because no allosteric modulators of malate synthase have been described to date. Preliminary docking studies on the well-known active site using FRED correctly placed malate in the malate synthase binding site, anchoring the molecule by strong ionic interactions between the carboxylate and $\mathrm{Mg}^{2+}$ and the hydrogen bonds formed with the surrounding water molecules.

Comparing Figure $5 \mathrm{a}$ and $5 \mathrm{~b}$, our docking results clearly suggests that bromopyruvate competes with malate for the substrate binding pocket of malate synthase and does not appear to interfere with coenzyme binding. We can therefore infer a simple competitive inhibition mechanism in our mathematical models. In addition to our cheminformatics analysis, using data retrieved from the literature, we identified and simulated four compound inhibitors of malate synthase and inhibitors of isocitrate lyase (ICL1 and ICL2). The inhibitors and their associated $\mathrm{K}_{\mathrm{I}}$ values are listed in Table 2 and Table 3, respectively.

\subsection{Metabolic Consequence of Malate Synthase and Isocitrate Lyase Inhibition in Mtb}

Emulating the in vitro Wayne NRP model, we simulate Mtb metabolism during growth in well-aerated, slow-stirred hypoxic, and vigorously agitated hypoxic conditions. These conditions result in exponential growth, exponential growth followed by NRP, and exponential growth followed by death, respectively. The vigorously agitated model is used as the negative control versus the well-aerated/non-inhibited system and the slow-stirred/ non-inhibited system, which represents our positive active growth control and our positive $\mathrm{NRP} /$ latent growth control. We simulate NRP Mtb growth using enzyme activity rates observed in empirical studies. Specifically we increase GDH (EC 1.4.1.10) ten fold, ICL 
(EC 4.1.3.1) four fold, and reduce MS (EC 2.3.3.9) two-fold [Wayne and Hayes, 1996; Smith, C., et al., 2003]. We compare our inhibition models for these systems.

3.2.1 Results of SCB analysis of Mtb TCA/Glyoxylate Bypass during inhibition of malate synthase-Using our theoretical model, we analyze the TCA/glyoxylate cycle in the absence of inhibitory molecules and during inhibition of MS by the molecular substrates identified in Table 2. The simulation employs a simple competitive inhibition model, where the $\mathrm{K}_{\mathrm{M}}$ is increased by $[\mathrm{I}] / \mathrm{K}_{\mathrm{I}}$ ([I] is the concentration of the inhibitor and $\mathrm{K}_{\mathrm{I}}$ is the inhibition constant). The simulation framework allows the incorporation of more complex inhibition models. Addition of each inhibitor is individually simulated and we compare the results to the non-inhibited controls. To enable comparison, we normalize results to the non-inhibited controls for the well-aerated, active growth model (Figure 6) or the slow-stirred, NRP growth model (Figure 7). Figures 6 and 7 show the effects of MS inhibition on components of the TCA/glyoxylate cycle during aerated growth and slowstirred/hypoxic growth where oxygen is slowly depleted from the system.

Figures $6 \mathrm{~A}-\mathrm{B}$ and $7 \mathrm{~A}-\mathrm{B}$ show the concentration of glyoxylate and glycine $(\mathrm{mM})$ over a tenday simulation period for each of the MS inhibitors, where the concentration of the inhibitor is $11.4 \mathrm{mM}$ which equals $100 \mathrm{x}$ the initial concentration of glyoxylate. In the figure legends the following abbreviations are used: control/non-inhibited (Non), bromopyruvate (Bromo), glycolate (Glyco), oxalate (Oxal), phosphoenol-pyruvate (Ppy). Simulation results from each inhibition model are normalized against the control, non-inhibited model for the respective environmental condition. To compare the impact of the various inhibitors, we calculate the average relative fold change by taking the mean of the $\log _{2}$ normalized concentration value for each metabolite. Figures $6 \mathrm{C}-\mathrm{F}$ and $7 \mathrm{C}-\mathrm{F}$ show the average relative fold difference for metabolites from the inhibited systems for simulation times greater than 40h; this time frame captures the tail end of logarithmic replication through both phases of NRP in the slow-stirred system [Wayne and Hayes, 1996]. In the graphs a value of zero indicates parity with the control, non-inhibited system. Figures $6 \mathrm{C}$ and $7 \mathrm{C}$ show the system for inhibitor concentration equal to $1 \mathrm{x}(0.114 \mathrm{mM})$ the concentration glyoxylate and $6 \mathrm{D}$ and 7D show the system for the 100x inhibition level. Both the aerated and slow-stirred simulation results show significant increase in glyoxylate and glycine levels when MS is inhibited, with the strongest inhibitor bromopyruvate resulting in the largest increase in the concentration of the metabolites. Additionally we observe a significant dosage-dependent increase between the 1x and 100x inhibition levels as clearly depicted in 6C-D and 7C-D.

Figures $6 \mathrm{E}-\mathrm{F}$ and $7 \mathrm{E}-\mathrm{F}$ show relative fold-change values for metabolites with less pronounced variations in the inhibited system, including malate, oxaloacetate, citrate, $\mathrm{NADH}$, and NAD. The metabolites directly downstream of the inhibited MS such as malate experience a net increase for some inhibitors in the aerated and for all inhibitors in the slowstirred systems. Increasing inhibitor levels to 100x results in an overall net decrease in malate, oxaloacetate, citrate and NADH. However there is a slight net increase in NAD relative to the control. For both the aerated and slow-stirred model the NAD increase does not result in a proportional decrease in NADH, and results in a disruption of the NAD:NADH ratio. Inhibition of MS in the aerated system resulted in a slightly greater change in glyoxylate and glycine than in the slow-stirred system. The opposite holds for 
metabolites downstream of MS, where the greater decrease occurs in the slow-stirred system.

\subsubsection{Results of Analysis of Mtb TCA/Glyoxylate Bypass during inhibition of isocitrate lyase-We investigate inhibition of ICL1 and ICL2 using the inhibitors listed} in Table 3. ICL1 and ICL2 catalyze the reaction ICIT $=G L X+S U C C$, a key biochemical process in the TCA cycle. As in the MS-inhibitor system we compare dosage response and the average $\log _{2}$ normalized concentration values for the aerated and slow-stirred inhibition models normalized against their respective non-inhibited controls. Figures 8A-D and 9A-D show the concentration of glyoxylate, glycine, malate, and oxaloacetate $(\mathrm{mM})$ over a ten-day simulation period for each of the ICL1/ICL2 inhibitors, where the concentration of the inhibitor is $6.0 \mathrm{mM}$, which equals $100 \mathrm{x}$ the initial concentration of isocitrate. In the figure legends the following abbreviations are used: control/non-inhibited (Non), 3-nitropropionate (Np3), bromopyruvate(Bromo), itaconic anhydride (Ith), itaconate (Ita). We observe notable inhibition of glyoxylate and glycine levels at the 100x inhibitor concentration for both the aerated and low oxygen models. There are observable inhibitor-mediated reduction of malate and oxaloacetate (Figures 8C-D and 9C-D) for both simulated conditions.

Figure $8 \mathrm{E}-\mathrm{H}$ and Figure 9E- $\mathrm{H}$ compare the average relative fold change at $1 \mathrm{x}$ and $100 \mathrm{x}$ inhibition levels using the normalized metabolite concentrations for the aerated and slow oxygen depletion conditions, respective. Fold changes in $8 \mathrm{E}-\mathrm{H}$ are for simulation times greater than $40 \mathrm{~h}$ and fold changes in $9 \mathrm{E}-\mathrm{H}$ are for simulation times greater than $3 \mathrm{~h}$ to capture the region with greater variation in malate and oxaloacetate for the slow-stirred system (Figure 9C and D). In general, we observe consistent correlation between the strength of the inhibitor and the inhibition impact on metabolic substrates, such that the strongest inhibitor leads to the greatest decrease for each metabolite. However we clearly see that at $1 \mathrm{x}$ inhibition levels we observe an unexpected net increase in the level of metabolites. This net increase becomes a net decrease with respect to the non-inhibited systems at the 100x inhibition level, demonstrating the impact of dosage on the system and potentially the impact of the presence of branched pathways and reaction rates on our ability to successfully produce a desired chemotherapeutic effect using targeted inhibition. We discuss this further in Section 4.

In comparing the results from Figure 8 and 9, we note that the inhibition of ICL1/2 has a greater impact on the slow-stirred/low-oxygen system than the well-aerated system. In Figures $8 \mathrm{~F}$ and $9 \mathrm{~F}$ we observe that ICL inhibition in the slow-stirred model results in a greater reduction of glycine than in the aerated system. The difference in relative glycine level occurs although both systems have comparable glyoxylate inhibition levels. We also observe lower levels of malate, oxaloacetate, citrate, NADH, and NAD in the slowstirred/NRP system than in the well-aerated system. These differences may correlate to the reduction in NADH/NAD recycling coupled to the reduced throughput of the glyoxylate to glycine shunt as a result of blocking ICL-mediated conversion of isocitrate to glyoxylate. The GtG shunt is more critical in the slow-stirred model as it mediates the conversion of NADH to NAD, which can help compensate for the oxygen-related reduction of $\mathrm{NADH} / \mathrm{NAD}$ recycling. 


\subsubsection{Analysis of Mtb TCA/Glyoxylate Bypass during combined MS and ICL}

inhibition-The goal of chemotherapeutic treatment that targets the pathogen is clearly the elimination of the pathogen. We use our theoretical model to investigate whether a treatment regimen that targets MS or ICL only versus one that targets both in combination is more effective at inhibiting the growth of active or NRP Mtb. To compare combined inhibition with results from our single-inhibition simulations, we run multiple-inhibition simulations that combine strong, medium, and weak inhibitors of MS and ICL at 100x concentration levels. In these simulations we treat the non-inhibited aerated model as our control and compare results to the non-inhibited slow-stirred/NRP system, which represents our persistence profile, and the non-inhibited rapid oxygen depletion model, which represents our death profile.

We normalize all simulation output, including the slow-stirred and rapid-oxygen depletion results, against our aerated control. We compare fold change among all conditions using the $\log _{2}$ of the mean normalized metabolite concentration value. Figure 10 shows the results of this study for glyoxylate, glycine, malate, oxaloacetate, citrate, NADH, and NAD for the aerated model (Figures 10A, C, E) and the slow-stirred model (Figures 10B, D, F) for simulation times greater than $40 \mathrm{~h}$. The figure legends represent the various inhibition conditions tested with the following abbreviations: strong, medium, and weak MS inhibitors represented by bromopyruvate(Bro), phosphoenol-pyruvate (Ppy), and glycolate(Gly), respective; strong, medium, and weak ICL inhibitors represented by 3-nitropropionate (Np3), itaconate (Ita), and itaconic anhydride (Ith), respective; non-inhibited slow-stirred (Slw) and rapid-oxygen depletion (Vig) systems.

In Figures 10A-B we observe that glycine levels are increased under environmental conditions that lead to Mtb persistence (4.1923 fold for Slw) and slightly increased for conditions that lead to Mtb death ( 0.0038 fold for Vig). Glyoxylate levels are increased during Mtb persistence (2.9213 fold for Slw) but decreased during Mtb death ( -0.2208 fold for Vig). During aerated growth, strong inhibition of ICL by Np3 most effectively reduces the levels of glycine and glyoxylate, reducing levels of these metabolites to well below that reached under rapid oxygen depletion. Combined inhibition of MS and ICL using a weak MS inhibitor (Gly) and Np3 reduced glycine and glyoxylate below the levels found in slowstirred models while strong MS inhibition significantly increased metabolite levels. Under slow-stirred conditions only strong ICL inhibition by Np3 reduced glycine and glyoxylate levels below that of persistent bacteria but not to the level of dying bacteria, suggesting a more effective inhibition strategy is needed to significantly reduce glyoxylate and reduce possible GtG shunt mediated generation of NAD.

Other than glycine and glyoxylate, the impact of inhibitors on most of the metabolites in the aerated model is less pronounced. In Figures $10 \mathrm{C}$ and $10 \mathrm{E}$ we plot the metabolites for the inhibited aerated system separately and include the Slw and Vig results in Figures 10D and $10 \mathrm{~F}$ with the slow stirred system where the fold changes are more pronounced. From Figure $10 \mathrm{D}$ we see that fold change between the control, non-inhibited aerated system is greater for malate, oxaloacetate, and citrate in the Vig system $(0.3138,-0.2667,-0.1813$, respective) than the Slw system $(0.1000,-0.0543,-0.0365$, respective). Under both conditions we see a positive fold change for malate and a negative fold change for the metabolites downstream 
of malate. The decrease in oxaloacetate and likely subsequent decrease may be attributed to reduced NAD levels ( -0.2793 and -3.6338 fold for Slw and Vig, respective) due to reduced oxygen-enabled recycling of NADH to NAD (Figure 10E), where NADH levels increased by 0.6549 and 1.3401 fold for Slw and Vig, respective. Under well-aerated conditions while the effects of inhibition are relatively minor, there is no increase in malate. Combined inhibition of MS and ICL by strong inhibitors (Bro/Np3) results in the most effective reduction of oxaloacetate and citrate. In general inhibition of the slow-stirred system slightly increases (in the case of malate and NADH) or slightly reduces (for oxaloacetate, citrate, and NAD) metabolite levels beyond that of persistent Mtb (Slw). While several inhibition strategies slightly edge the slow-stirred system towards levels found in the Vig system, the combination of weak MS inhibition and weak ICL inhibition (Gly/Ith) has a slight advantage over the alternative strategies.

\subsubsection{Analysis of Mtb adaptive response during ICL inhibition by 3-}

nitropropionate-An expected consequence of drug therapy is the initiation of an adaptive response by the pathogen to the chemotherapeutic agent. In an extensive study by Boshoff et al., the genetic response of Mtb to various inhibitory molecules and environmental stresses was investigated (Boshoff et al., 2004). While the study did not include response to specific inhibitors considered in our model, molecules that inhibit enzymes involved in the TCA cycle and the electron transport chain were reported. Results from the study indicate that triclosan (TRC), a broad-spectrum antibacterial molecule, inhibits bacterial respiratory enzymes, including inhibition of succinate dehydrogenase. While we are not directly targeting succinate dehydrogenase in our SCB model, as simulation results suggest inhibition of isocitrate lyase can negatively impact the bacterial respiratory system. In response to TRC inhibition, Mtb up-regulates genes in various pathways including genes involved in the tricarboxylic acid cycle. In supplemental gene expression (GEO GSE1642) data provided by Boshoff et al., citrate synthase ( $\mathrm{gltAl/}$ Rv1131) is increased 1.73 fold six hours following the addition of 50ug of TRC.

Ebel et al., studied the direct consequence of isocitrate lyase inhibition by 3-nitropropionate in Aspergillus fumigatus (Ebel et al., 2006). In response to inhibition, A. fumigatus increased expression of the icl promoter in a linear, concentration-dependent manner: $\mathrm{y}=72.825(\mathrm{x})+$ 395.97, where $\mathrm{x}$ represents the concentration 3-nitropropionate and $\mathrm{y}$ is the specific activity of the icl promoter-coupled $\beta$-galactosidase reporter protein. We normalize the equation using the baseline specific activity of the reporter protein in the absence of the inhibitor (395.97) and calculate the relative change in $i c l$ expression during inhibition. In the presence of $6 \mathrm{mM}$ 3-nitropropionate, which is the level of inhibitory substrate used in our SCB models, we estimate a 2.1 fold increase in isocitrate lyase expression, two-hours postinhibition in concurrence with sample collection times reported (Ebel et al., 2006).

We investigate the metabolic consequence of Mtb response to indirect inhibitor-induced respiratory stress and direct inhibition of isocitrate lyase using data from the Boshoff et al., and Ebel et al., studies, respective. In Figure 11 we compare the metabolic consequence of Mtb adaptation to inhibition of isocitrate lyase by 3-nitropropionate for the aerated (Fig. 11A,B) and NRP/slow-stirred system (Fig. 11C,D) to our previous results from the inhibition models. For glyoxylate and glycine, the two metabolites most impacted by 
inhibition, we note that the adaptive response of Mtb attempts to counteract the effect of inhibition resulting in an increase in both metabolites. However the adaptive responses included in our model did not significantly reverse the inhibition phenotype, which is clearly seen when comparing the results to the non-inhibited models (inset figures).

\section{Discussion}

In this work we demonstrate the feasibility of using computational systems chemical biology methods to study the interaction of possible small-molecule inhibitors with Mtb's malate synthase and isocitrate lyase, key enzymes that enable the bacterium to persist under oxidative stress conditions. SCB-related virtual screening provides insight into possible mechanisms of inhibition using docking-based studies of small-molecule displacement of substrates in the binding pockets of the enzyme. Docking-based screening can help refine inhibitor candidates and identify molecules capable of disrupting enzymatic activity. Further work is needed to effectively quantify and directly link screening results to inhibition parameters for SCB simulation studies.

The major challenge of the proposed systems chemical biology approach is in creating a strong link between the systems biology model and the cheminformatics counterpart. As discussed in this work, the traditional bottom-up systems biology model is composed of equations that quantify and describe the biochemical outcome of inhibiting Mtb's TCA and glyoxylate bypass pathway when using known enzymatic inhibitors and previously identified inhibition constants. However, such a model does not include the chemical information that is required when planning novel molecules or analogues with the same aim. Our first attempt to address this problem consisted of the study of two key enzymes (ICL and MS) and their inhibitor molecules using docking, a fast approach that is commonly used in the initial steps of the virtual screening step. We have shown results of inhibitors docked into the active site of the malate synthase in order to understand the chemical interactions between the ligands and the macromolecule in comparison with the X-ray crystallographic structure (Fig. 5). Docking results can enable the prediction of novel molecules as putative enzymatic inhibitors for further SCB analysis. As seen in Fig. 5b, it is easily perceived that the bromopyruvate molecule is a competitive inhibitor that binds into the substrate site and that it does not cause any perturbation to the coenzyme. Results of the docking study provide an important structural piece of information that supports the proposed mode of action and also provides valuable mechanistic data directly linking our cheminformatics workflow and our systems biology model.

Usually the docking program gives reasonable poses, but the main drawback of this approach is the scoring function (Warren 2006, Huang 2010). This limitation impairs confident quantitative analyses of the energetics involved in the system and allows, at best, semi-quantitative (or comparative) analyses pointing to a trend in affinity/inhibition of a set of molecules. One possible alternative to this approach could be the molecular modeling of the system by means of molecular dynamics (MD). Nonetheless, MD is not yet designed for a fast trial of the chemical space like docking and it is often applied on further steps of the drug discovery pipeline, when seeking the influence of more subtle chemical modifications of new derivatives (Durrant 2011). Even if a more complex approach like the Free Energy 
Perturbation could be applied to the system and $\Delta \mathrm{G}$ would be determined, it would not always be straightforward to link $\mathrm{K}_{\mathrm{a}}$ (or $\mathrm{K}_{\mathrm{d}}$ ) to $\mathrm{K}_{\mathrm{i}}$.

With the goal of disrupting Mtb's ability to survive, results from Section 3.2 provide insight regarding key consequences of MS and ICL inhibition. First, the results of MS versus ICL inhibition under common environmental conditions suggest that inhibition of MS has the greatest metabolic impact on glyoxylate versus malate and downstream metabolites. However ICL targeting impacts both glyoxylate and malate due to the branching architecture of the system, which enables multiple pathways from isocitrate to malate production. Therefore for similar conditions, ICL is more consequential at reducing levels of malate, which can limit malate mediated gluconeogenesis-based production of NAD. Additionally ICL inhibition can lead to reduction in oxaloacetate available for amino acid biosynthesis.

Our second observation based on our model is that variations in the microenvironment impact the effectiveness of inhibition on malate and downstream metabolites but has less of an impact on glyoxylate. For both MS and ICL inhibition models, the magnitude of the relative fold change for malate, oxaloacetate, citrate, NADH and NAD increase under slowstirred/NRP conditions; this may be related to the reduction of oxygen-mediated NAD recycling in the NRP system compounding the effect of the inhibitor. Inhibition disrupts both systems' NAD:NADH ratio, the net effect being lower NADH levels and slightly increased NAD levels. Under NRP, the GtG shunt activity increases ten fold, further depleting NADH to produce NAD. Since NAD availability impacts reactions relevant for malate and oxaloacetate production we observe a greater variation in these metabolites during MS inhibition when the alternative pathways to malate production require NAD to effectively function.

Our third observation from the combined inhibition model is that targeting ICL directly impacts the glyoxylate to glycine shunt and limits Mtb's ability to use this alternative pathway to replenish NAD. By comparing inhibitor models to the three non-inhibited model, results suggest that a strong ICL inhibitor is the more effective treatment to move the metabolic profile of persistent Mtb towards that of dying Mtb represented by the vigorously agitated model. While we significantly reduced glyoxylate and glycine using Np3, more effective strategies are needed to significantly impact other metabolites such as oxaloacetate and NAD co-factor.

Lastly, our SCB study of malate synthase and isocitrate inhibition demonstrates the complex interplay between the architecture and kinetics of a biochemical network and results of system inhibition. In addition to the bacterium's use of alternative pathways to compensate for inhibited reactions, the kinetics of the system likely contributes to unanticipated outcomes. In addition to variations due to the strength of the inhibitor, the system response at $1 \mathrm{x}$ versus 100x yielded different outcomes. During ICL inhibition (Figures 8 and 9) at the $1 \mathrm{x}$ level there is a net increase of all metabolites except NAD and at the 100x inhibition level we observe a net decrease for all metabolites except NAD, which has a small net increase. The opposing behavior at $1 \mathrm{x}$ versus $100 \mathrm{x}$ is partly due to isocitrate functioning as a branch point in our TCA cycle model and the relative kinetic rates of the reactions that 
connect isocitrate and malate. If we consider for example the increase in glyoxylate at low inhibitor concentrations, we postulate that during ICL inhibition the systems ability to use alternate NAD and FAD dependent reactions to produce succinate and ultimately malate may lead to comparatively higher levels of malate than glyoxylate, making the inverse malate to glyoxylate reaction more favorable. The reaction rates associated with these reactions provide some support for this argument. The forward Vmax for malate synthase is $0.3333 \mathrm{mM} / \mathrm{s}$ and inverse reaction rate for the production of glyoxylate from malate is $0.0033 \mathrm{mM} / \mathrm{s}$. The ICL1/2 reaction rates are comparable to the inverse MS rates, with the forward Vmax equal to $0.0049 \mathrm{mM} / \mathrm{s}$ and $0.0065 \mathrm{mM} / \mathrm{s}$, respect. With [Glyoxylate] $\cong$ $3 \mathrm{e}^{-6} \mathrm{mM}$ and [Malate] $\cong 1.84 \mathrm{e}^{-2}$, this increases the favorability of the reverse reaction. Furthermore the Vmax of isocitrate dehydrogenase 1/2 (ICD1/2), which converts isocitrate to alpha-ketoglutarate (AKG) is two-orders of magnitude greater than ICL1/2 (rates for ICD1/2 are $0.17 \mathrm{mM} / \mathrm{s}$ and $0.166 \mathrm{mM} / \mathrm{s}$, respective).

Finally as an extension of our SCB studies, we consider how an adaptive response by the bacteria to inhibitory or respiratory stress counteracts the metabolic impact of inhibition in the well-aerated and NRP system. We found that while notable increases in metabolites such as glyoxylate and glycine occur, the original non-inhibited levels were not reached. These results demonstrate the need to consider not only the interaction of a small-molecule and the target enzyme-reaction subsystem, but also the consequential response of the bacterial system that works to reverse the effects of chemotherapeutic agents.

SCB analysis of pertinent pathogen biochemical systems can ultimately enable us to study potential side effects of small-molecule perturbations on target and non-target pathways for various types of inhibitors, dosage levels, and micro-environmental conditions. As we demonstrate in this work, inhibition can result in unexpected system response due to structural and dynamic properties of the network. Use of computational SCB tools and methods can advance in silico tools used to support identification and predictive characterization of candidate small molecules. Insights from SCB based studies can aid in the development of novel therapeutics for challenging diseases like latent TB infection or potentially identify new uses for existing chemotherapies in the treatment of disease.

\section{Acknowledgments}

We would like to thank the reviewers for their helpful comments and suggestions for improving the initial version of our manuscript.

E.E.May partially supported by: a Department of Energy Laboratory Directed Research Grant from Sandia National Laboratories (Sandia is a multiprogram laboratory operated by Sandia Corporation, a Lockheed Martin Company for the United States Department of Energy's National Nuclear Security Administration under contract DEAC04-94AL85000); award number K25HL075105 from the NIH National Heart, Lung, and Blood Institute. T. Oprea and A. Leitao partially supported by a NIH U54 grant, award number U54-MH084690. A. Leitao was supported by Sao Paulo Research Foundation (FAPESP) project number 2011/07025-3.The content is solely the responsibility of the authors and does not necessarily represent the official views of the National Heart, Lung, and Blood Institute or the National Institutes of Health. 


\section{References}

Belta, C.; Finin, P.; Halasz, A.; Kim, MIJ.; Kumar, V.; Rubin, H. Modeling and analysis of metabolism and the stringent response in Mycobacterium tuberculosis. DARPA BioComp Proceedings; 2003.

Bentrup K, Miczak A, Swenson D, Russell D. Characterization of activity and expression of isocitrate lyase in Mycobacterium avium and Mycobacterium tuberculosis. Jrnl of Bacteriology. 1999; 181(23):7161-7167.

Beste D, Hooper T, Stewart G, Bonde B, Avignone-Rossa C, Bushell M, Wheeler P, Klamt S, Kierzek A, McFadden J. GSMN-TB: a web-based genome-scale network model of Mycobacterium tuberculosis metabolism. Genome Biology. 2007:8.

Boshoff H, Myers T, Copp T, McNeil M, Wilson M, Barry C. The Transcriptional Responses of Mycobacterium tuberculosis to Inhibitors of Metabolism. Jrnl Biological Chemistry. 2004; 239(78): 40174-40184.

Boström J, Greenwood JR, Gottfries J. Assessing the performance of OMEGA with respect to retrieving bioactive conformations. J Mol Graphics and Mod. 2003; 21(5):449-462.

Cosma CL, Sherman DR, Ramakrishnan L. The secret lives of the pathogenic mycobacteria. Annu Rev Microbiol. 2003; 57:641-676. [PubMed: 14527294]

Durrant JD, McCammon JA. Molecular dynamics simulations and drug discovery. BMC Biology. 2011; 9(71)

Ebel F, Schwienbacher M, Beyer J, Heesemann J, Brakhage A, Brock M. Analysis of the regulation, expression, and localisation of the isocitrate lyase from Aspergillus fumigatus, a potential target for antifungal drug development. Fungal Genetics and Biology. 2006; 43(7):476-489. [PubMed: 16603391]

Fang X, Wallqvist A, Reifman J. A systems biology framework for modeling metabolic enzyme inhibition of Mycobacterium tuberculosis. BMC Systems Biology. 2009; 3(92)

Fang X, Wallqvist A, Reifman J. Modeling synergistic drug inhibition of Mycobacterium tuberculosis growth in murine macrophages. Mol BioSyst. 2011; 7:2622-2636. [PubMed: 21713281]

Hawkins PCD, Skillman AG, Warren GL, Ellingson BA, Stahl MT. Conformer Generation with OMEGA: Algorithm and Validation Using High Quality Structures from the Protein Databank and Cambridge Structural Database. J Chem Inf Model. 2010; 50:572-584. [PubMed: 20235588]

Hawkins PCD, Nicholls A. Conformer Generation with OMEGA: Learning from the Data Set and the Analysis of Failures. J Chem Inf Model. 2012; 52:2919-2936. [PubMed: 23082786]

Jasmer RM, Nahid P, Hopewell PC. Clinical practice. Latent tuberculosis infection. N Engl J Med. 2002; 347(23):1860-6. [PubMed: 12466511]

May E, Schiek R. BioXyce: An engineering platform for the study of cellular systems. IET Systems Biology. 2009:3.

May, EE. Circuit-based Models of Biomolecular System Dynamics. In: Li, Peng; Silveira, Luís Miguel; Feldmann, Peter, editors. Simulation and Verification of Electronic and Biological Systems. Springer; 2011. p. 137-156.

McGann M, Almond HR, Nicholls A, Grant JA, Brown FK. Gaussian Docking Functions. Biopolymers. 2003; 68(1):76-90. [PubMed: 12579581]

Mdluli K, Spigelman M. Novel targets for tuberculosis drug discovery. Curr Opin Pharmacol. 2006; 6(5):459-467. [PubMed: 16904376]

Nelson, DL.; Cox, MM. Lehninger Principles of Biochemistry. 4. W. H. Freeman and Company; New York: 2005.

Oprea TI, May EE, Leitao A, Tropsha A. Computational systems chemical biology. Meth Molec Biol. $2011 ; 672: 459-488$.

Oprea TI, Tropsha A, Faulon JL, Rintoul MD. Systems chemical biology. Nat Chem Biol. 2007; 3(8): 447-450. [PubMed: 17637771]

Scheer M, Grote A, Chang A, Schomburg I, Munaretto C, Rother M, Söhngen C, Stelzer M, Thiele J, Schomburg D. BRENDA, the enzyme information system in 2011. Nucleic Acids Res. 2011; 39:670-676. 
Schnappinger D, Schoolnik G, Ehrt S. Expression profiling of host pathogen interactions: how Mycobacterium tuberculosis and the macrophage adapt to one another. Microbes and Infection. 2006; 8:1132-1140. [PubMed: 16517202]

Singh S, Ghosh I. Kinetic modeling of tricarboxylic acid cycle and glyoxylate bypass in Mycobacterium tuberculosis, and its application to assessment of drug targets. Theor Bio and Med Model. 2006:3. [PubMed: 16426453]

Smith CV, Huang C-c, Miczak A, Russell DG, Sacchettini JC, Bentrup KH. Biochemical and Structural Studies of Malate Synthase from Mycobacterium tuberculosis. J Biol Chem. 2003; 278(3):1735-1743. [PubMed: 12393860]

Warren GL, Andrews CW, Capelli AM, Clarke B, LaLonde J, Lambert MH, Lindvall M, Nevins N, Semus SF, Senger S, Tedesco G, Wall ID, Woolven JM, Peishoff CE, Head MS. A critical assessment of docking programs and scoring functions. J Med Chem. 2006; 4:5912-5931. [PubMed: 17004707]

Wayne L, Hayes L. An in vitro model for sequential study of shiftdown of Mycobacterium tuberculosis through two stages of non- replicating persistence. Infect Immun. 1996; 64:20622069. [PubMed: 8675308]

Wayne L, Lin K. Glyoxylate metabolism and adaptation of Mycobacterium tuberculosis to survival under anaerobic conditions. Infect Immun. 1982; 37:1042-1049. [PubMed: 6813266]

Wayne L, Sohaskey C. Nonreplicating persistence of mycobacterium tuberculosis. Annu Rev Microbiol. 2001; 55:139-63. [PubMed: 11544352] 


\section{Highlights}

- $\quad$ SCB related virtual screening and docking studies can provide mechanistic insight on inhibition.

- Unlike glyoxlate response, inhibitor impact on malate seems microenvironment dependent.

- For similar microenvironments, ICL versus MS inhibition is metabolically more consequential.

- Strong ICL inhibition causes metabolite levels in persistent Mtb towards levels in non-viable Mtb.

- $\quad$ SCB points to a complex interplay between network structure, kinetics, and therapeutic outcome. 


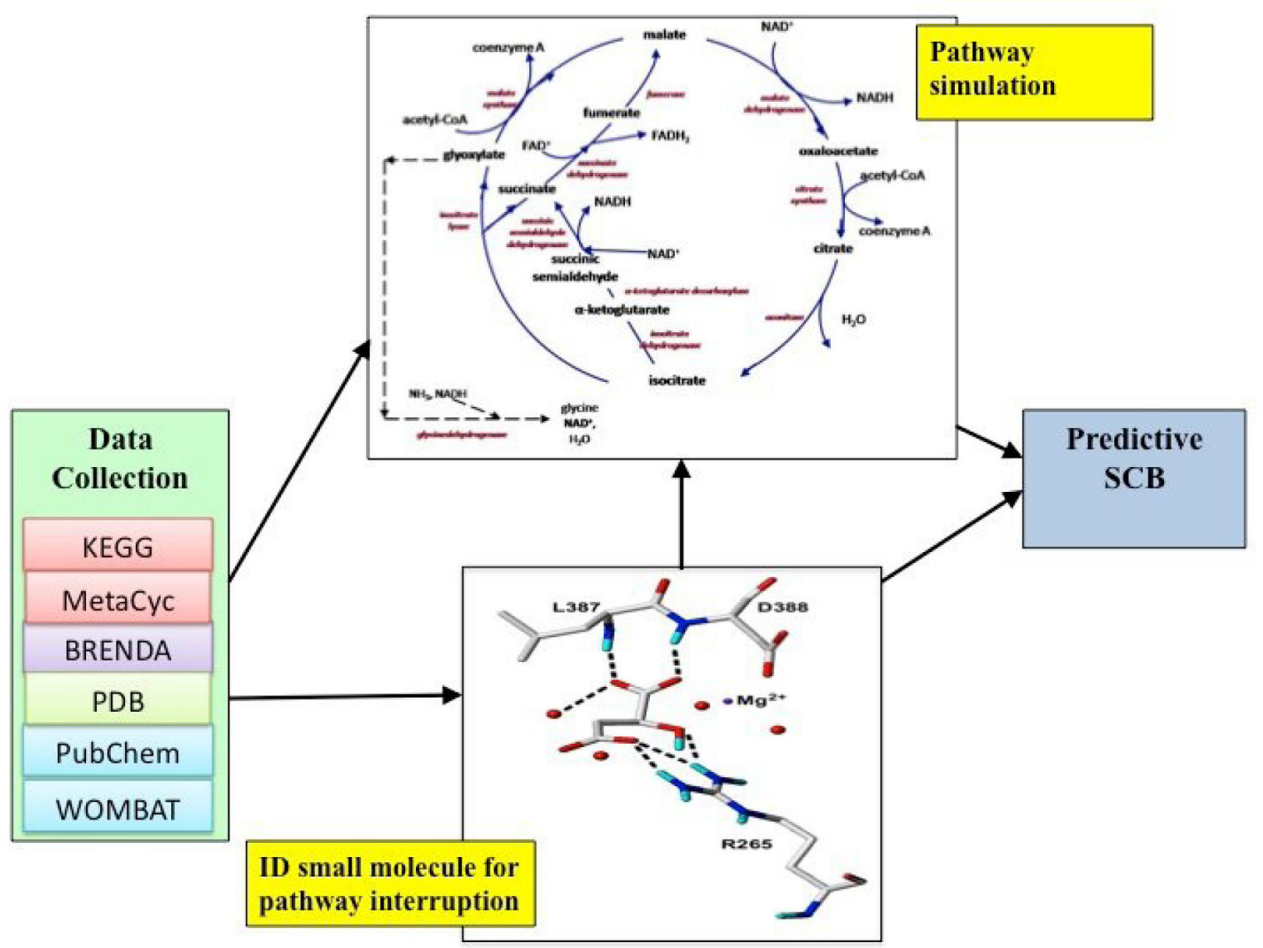

Figure 1.

Computational Systems Biology Workflow. 


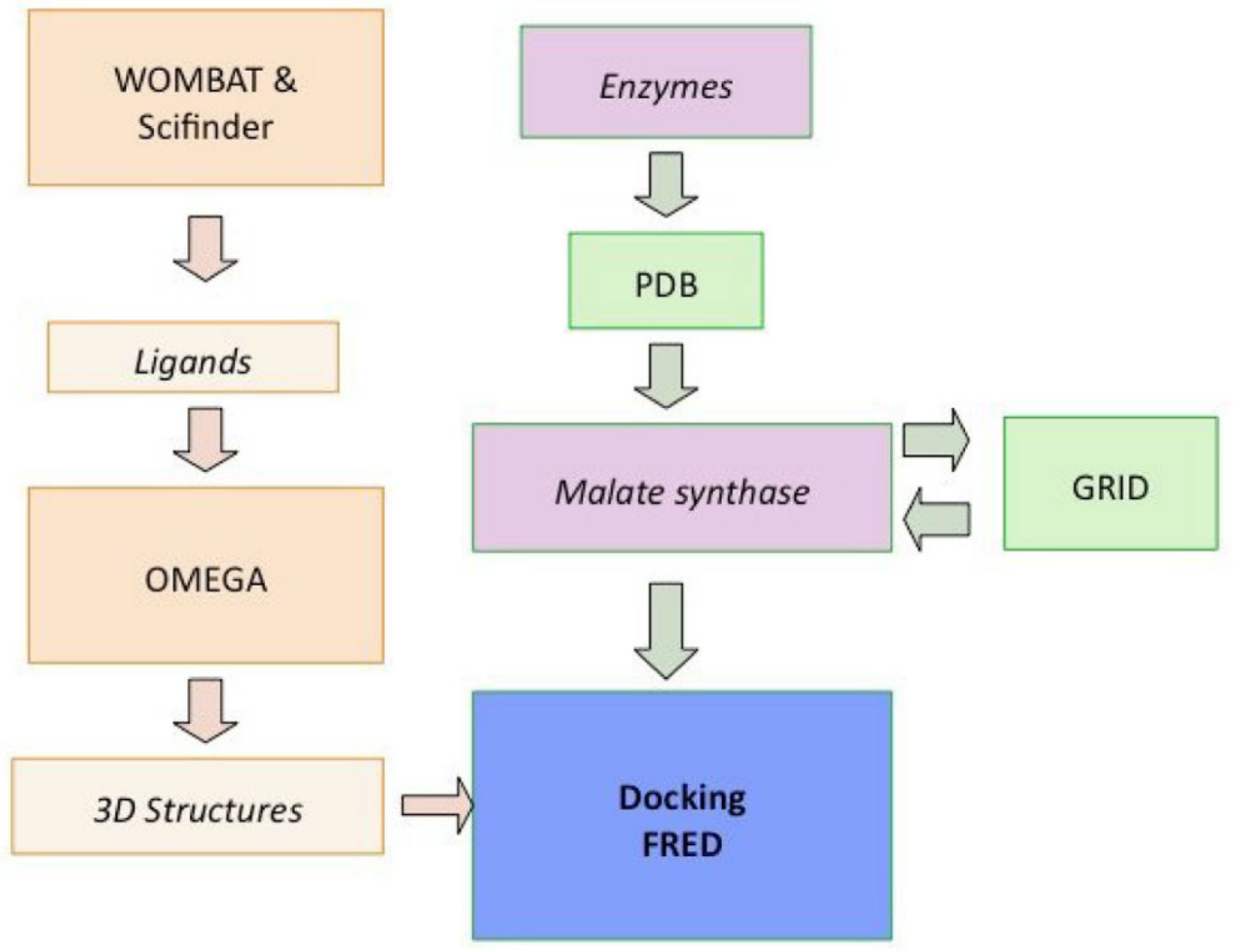

Figure 2.

Cheminformatics virtual screening workflow. 


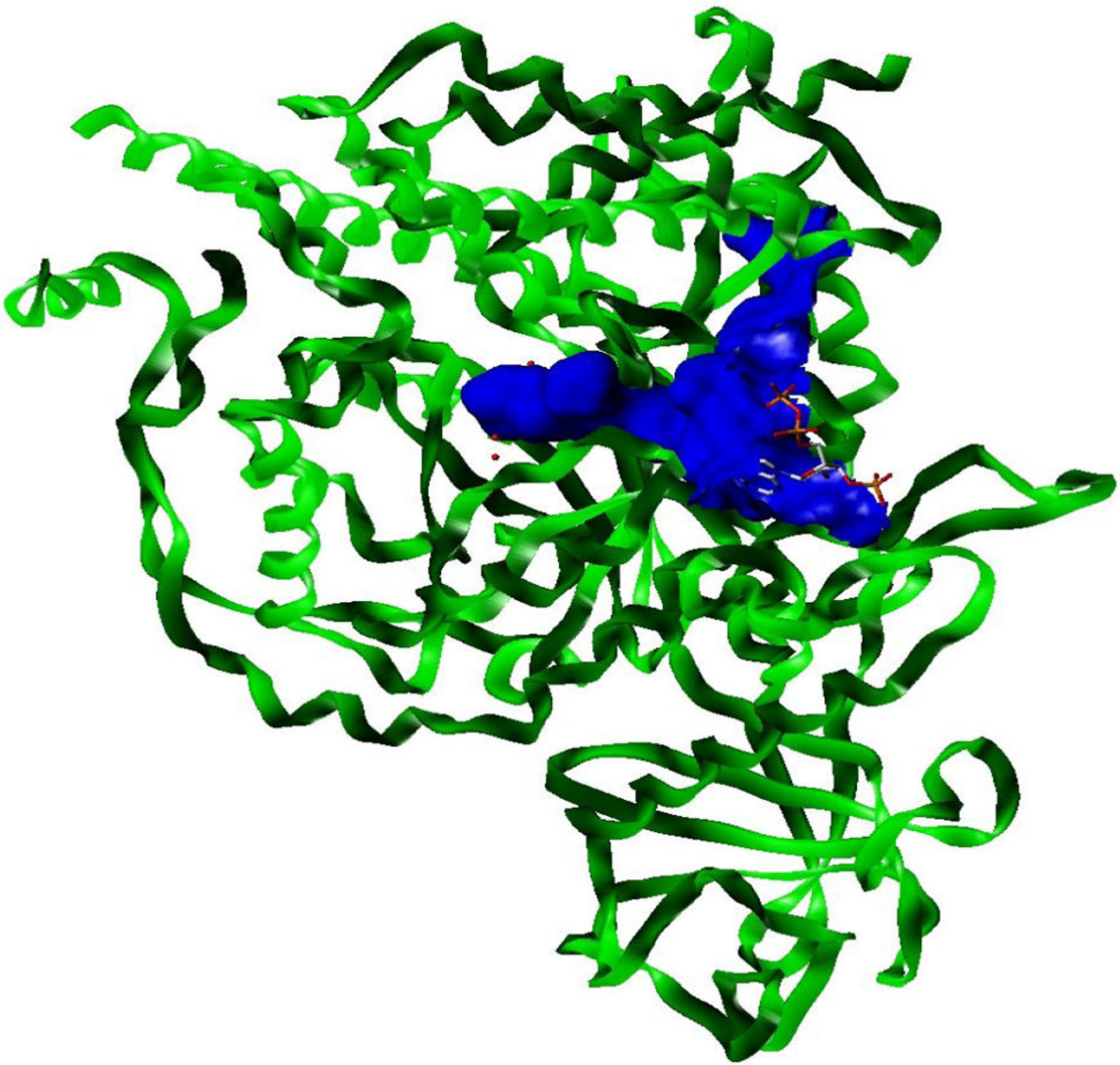

Figure 3.

Structural overview of the enzyme malate synthase (PDB code: 2GQ3) showing the substrate and cofactor pockets (blue surface). Enzyme is shown in green ribbons. 


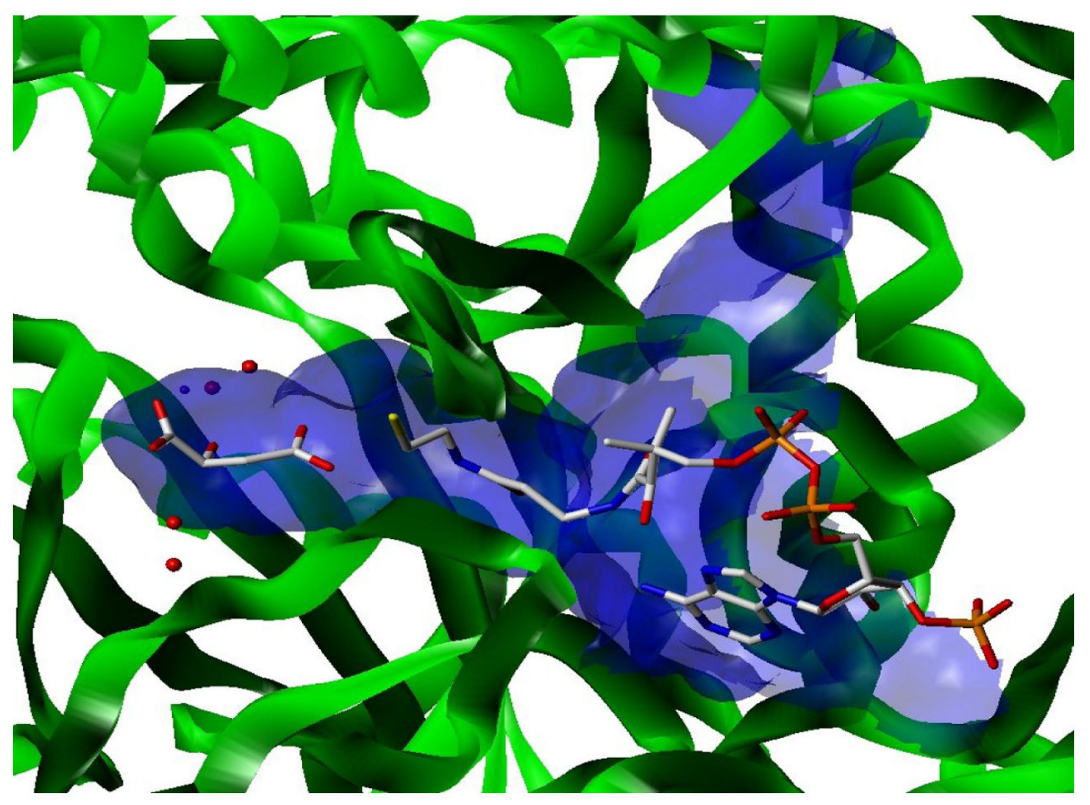

Figure 4.

Translucent view of the binding pockets surface of malate synthase showing malate (left), coenzyme A (right), water molecules and magnesium (spheres). Part of the CoA side chain is pointing outward. 


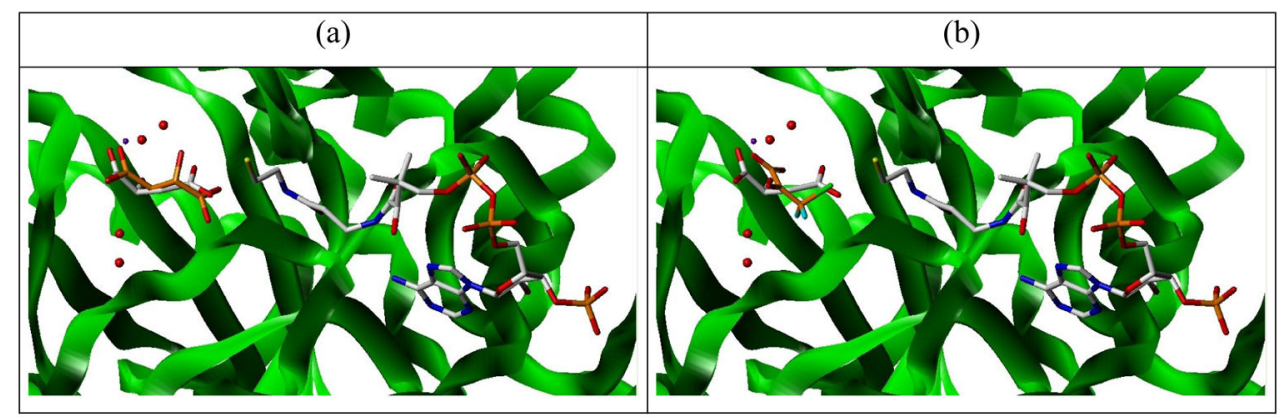

Figure 5.

Superposition of malate (a) and bromopyruvate (b) from the crystalographic X-rays (gray carbons) and the docking result (carbons in orange) into the malate synthase. 

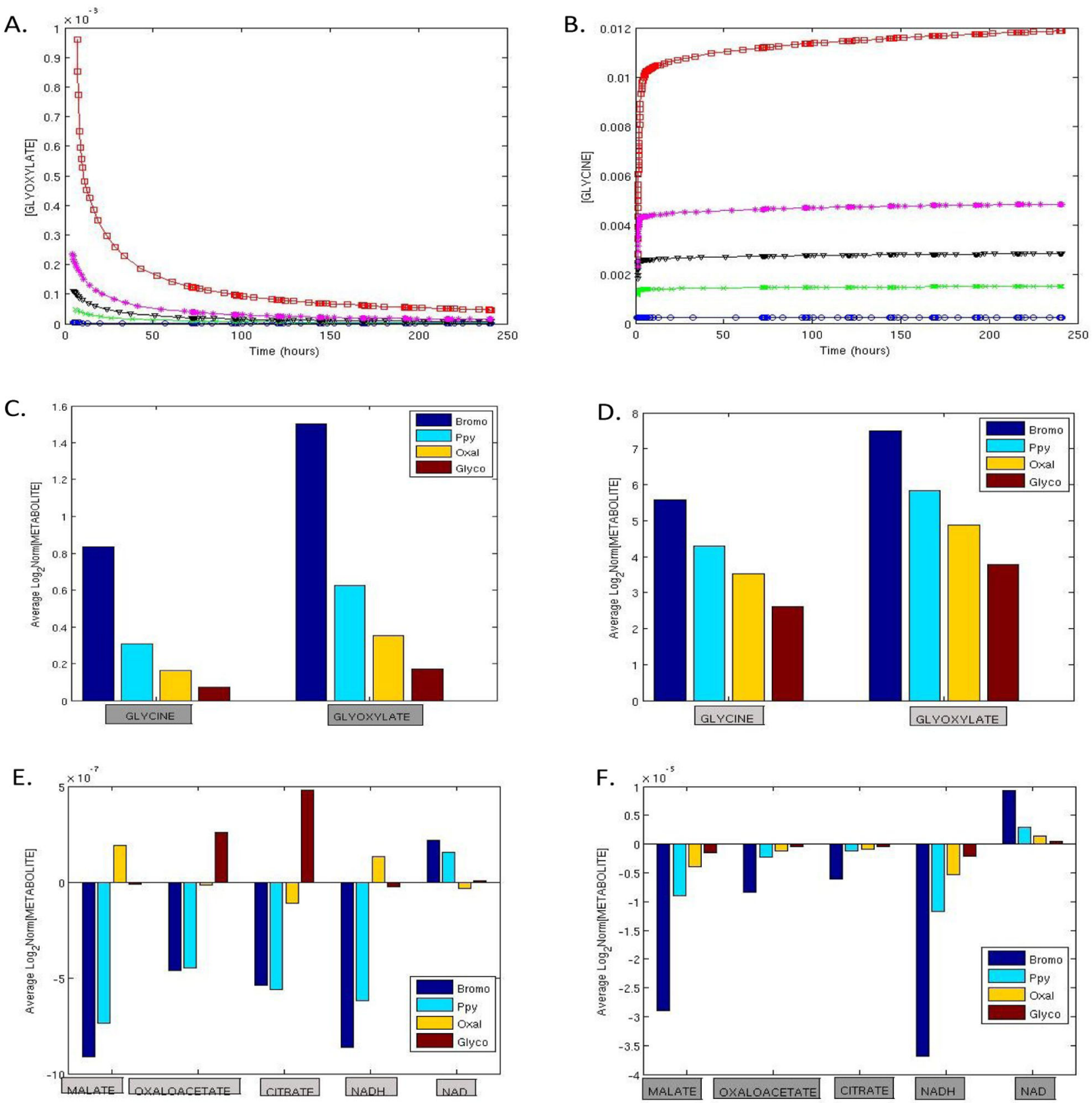

Figure 6.

Simulation results for SCB analysis of malate inhibition of Mtb during aerated growth. Greatest impact of MS inhibition on glyoxylate and glycine concentration for inhibitor levels at 100x the initial concentration of glyoxylate (A and B; legend - $o$ Non, -square-Bromo, - $x$-Glyco, -inverted triangle-Oxal, -star-Ppy). Impact of inhibitor observed but less pronounced for malate, oxaloacetate, citrate, NADH, and NAD. Dosage dependent difference observed for 1x (C, E) versus 100x inhibitor concentration (D, F). 
A.

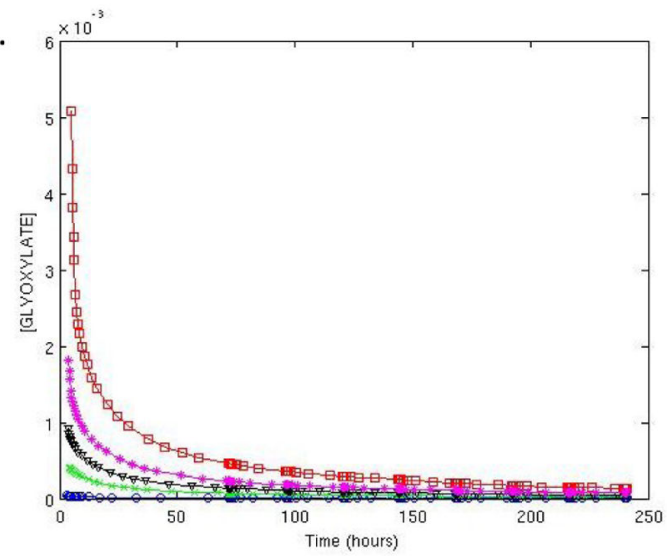

c.

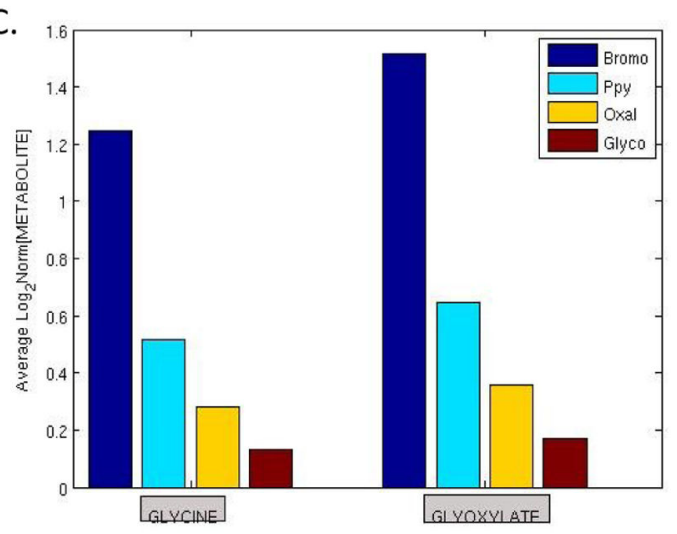

E.

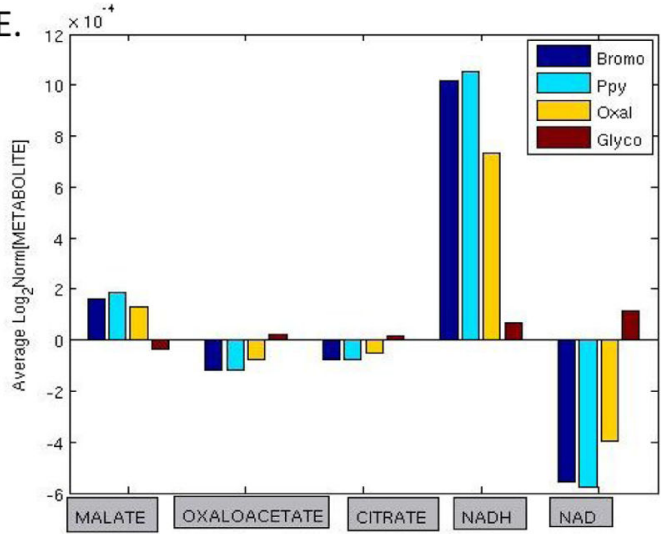

B.

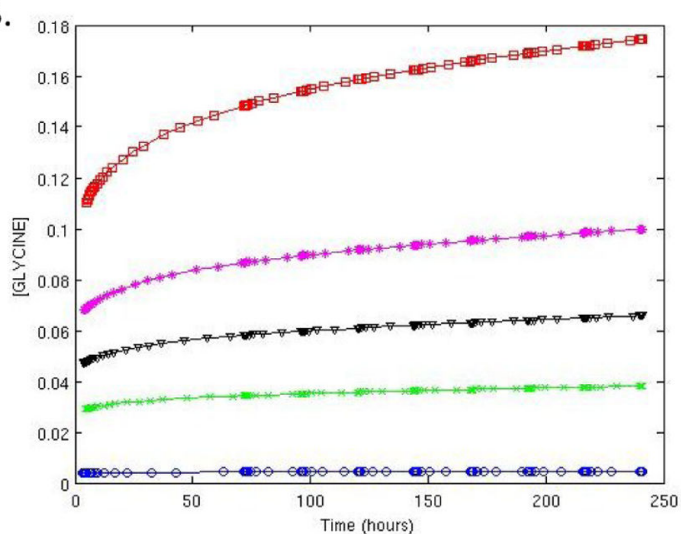

D.

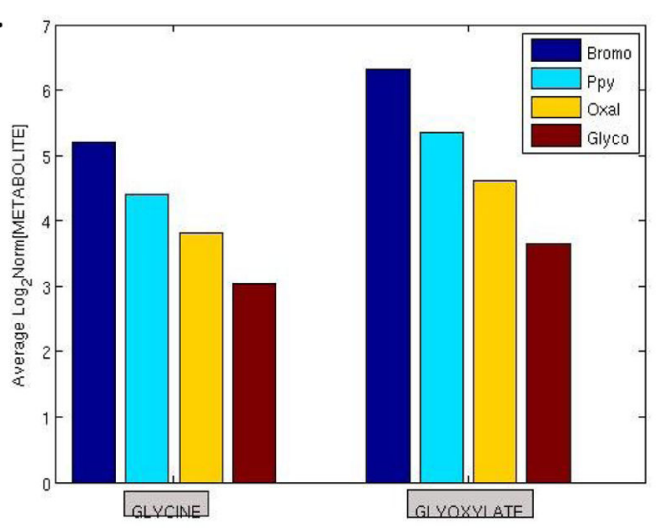

F.

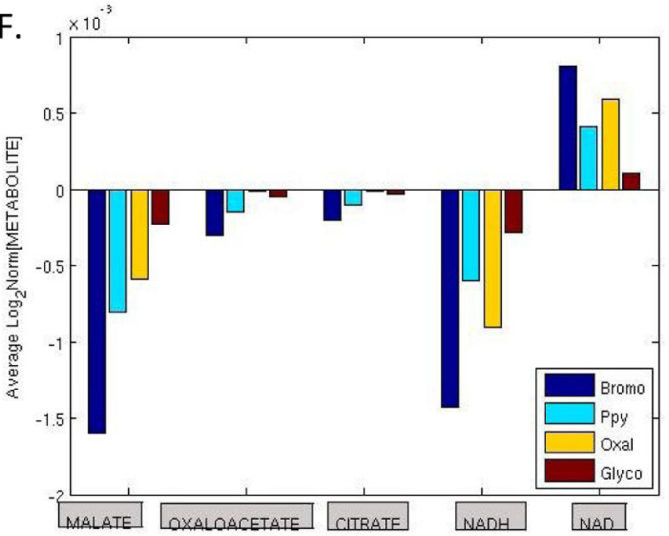

Figure 7.

Simulation results for SCB analysis of malate inhibition of Mtb during aerated growth. During slow O2 depletion, greatest impact of MS inhibition is on glyoxylate and glycine concentration for inhibitor levels at 100x the initial concentration of glyoxylate (A and B; legend -o-Non, -square-Bromo, -x-Glyco, -inverted triangle-Oxal, -star-Ppy). Impact of inhibitor observed but less pronounced for malate, oxaloacetate, citrate, NADH, and NAD. Dosage-dependent difference observed for 1x $(\mathrm{C}, \mathrm{E})$ versus 100x inhibitor concentration (D, F). 

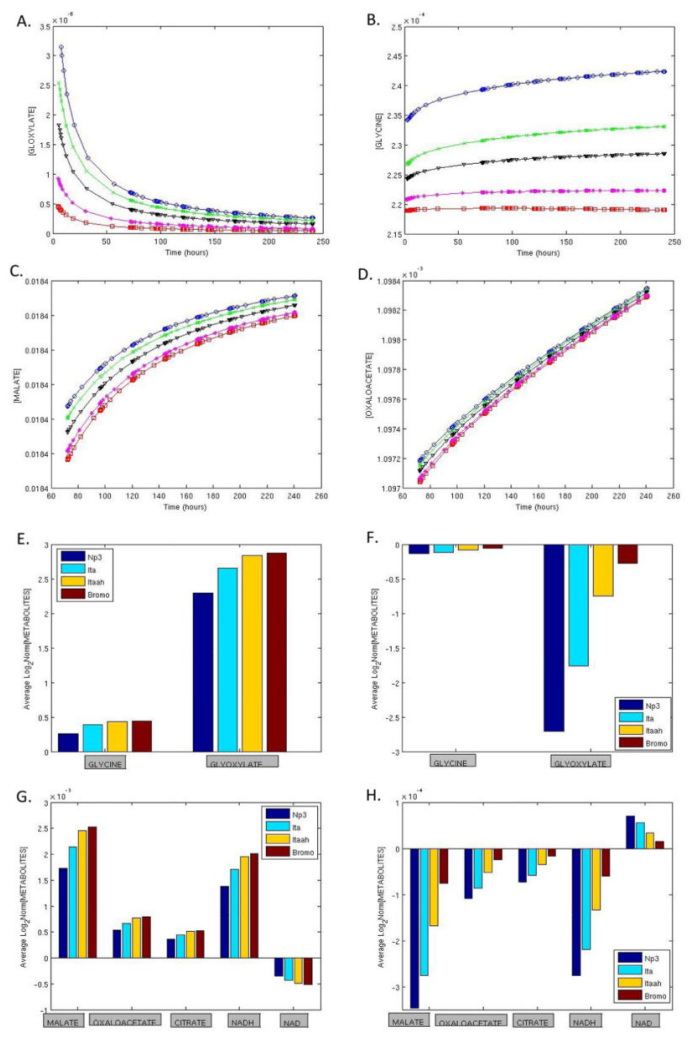

Figure 8.

During well aerated growth, ICL inhibition at 100x the initial concentration of isocitrate impacts several metabolites, with significant impact on glyoxylate(A), glycine (B), and notable reduction on malate (C) and oxaloacetate (D). (A-D legend -oNon, -square-Np3, -x-Bromo, -inverted triangle-Itaah, -star-Ita). Figures E-G compare dosage dependent impact, 1x (E,G) and 100x (F,H). 
A.

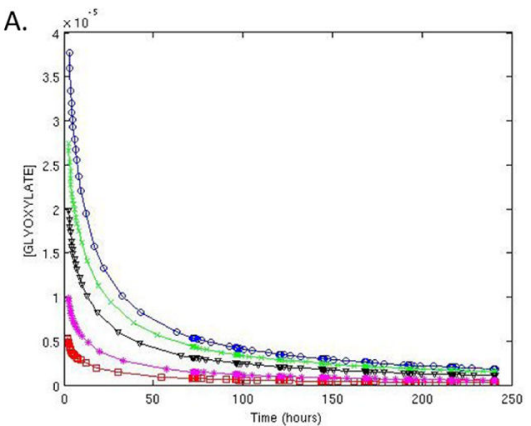

C.

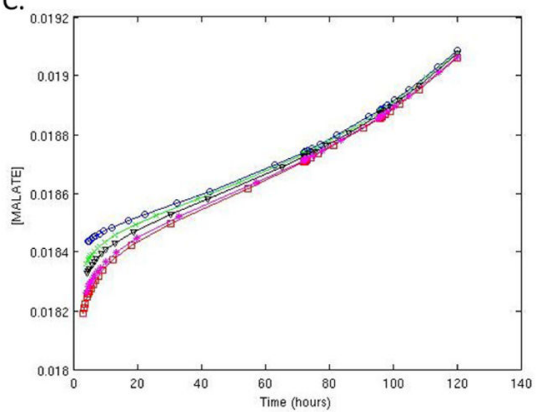

E.
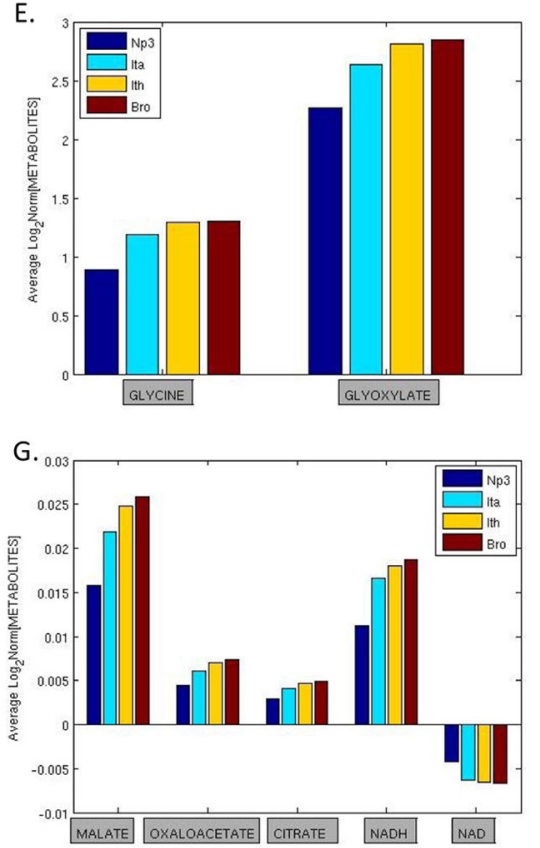

B.

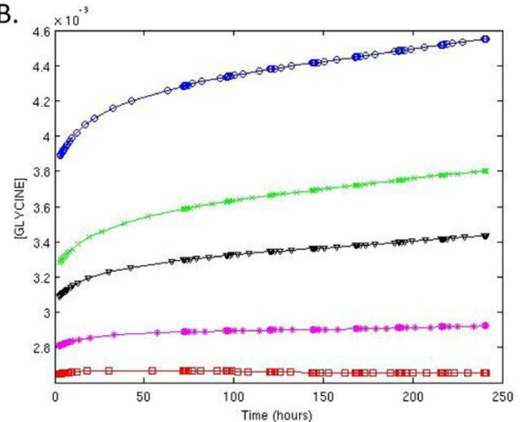

D.
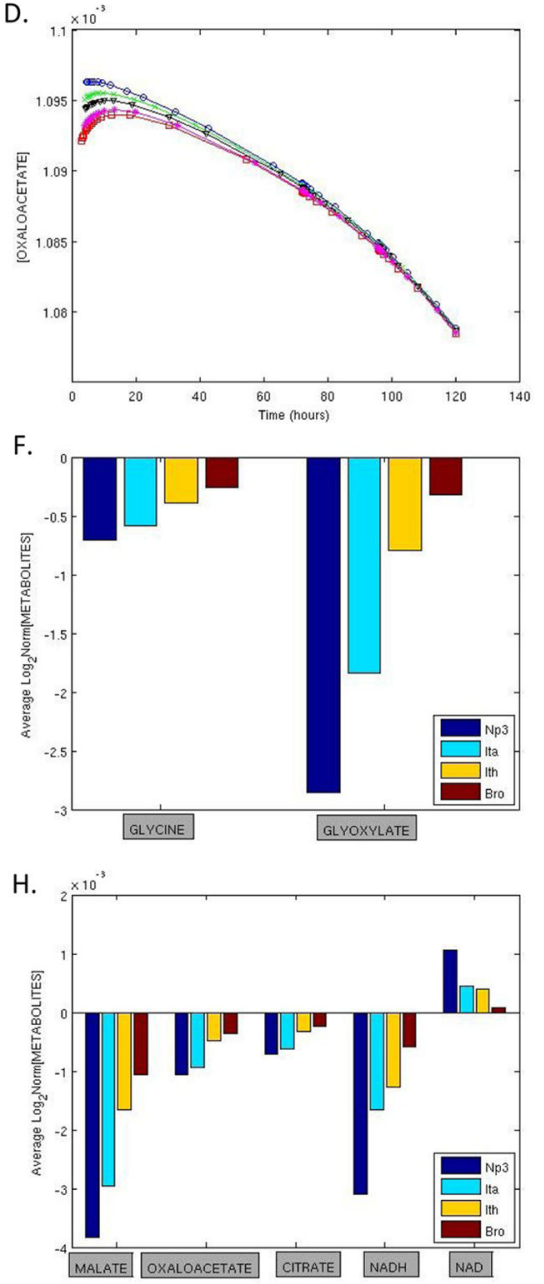

Figure 9.

During slow $\mathrm{O} 2$ depletion, ICL inhibition at 100x the initial concentration of isocitrate impacts several metabolites, with significant impact on glyoxylate(A), glycine (B), and notable reduction on malate (C) and oxaloacetate (D). (A-D legend - oNon, -square-Np3, -x-Bromo, -inverted triangle-Ith, -star-Ita). Figures E-G compare dosage dependent impact, 1x (E,G) and 100x (F,H). 

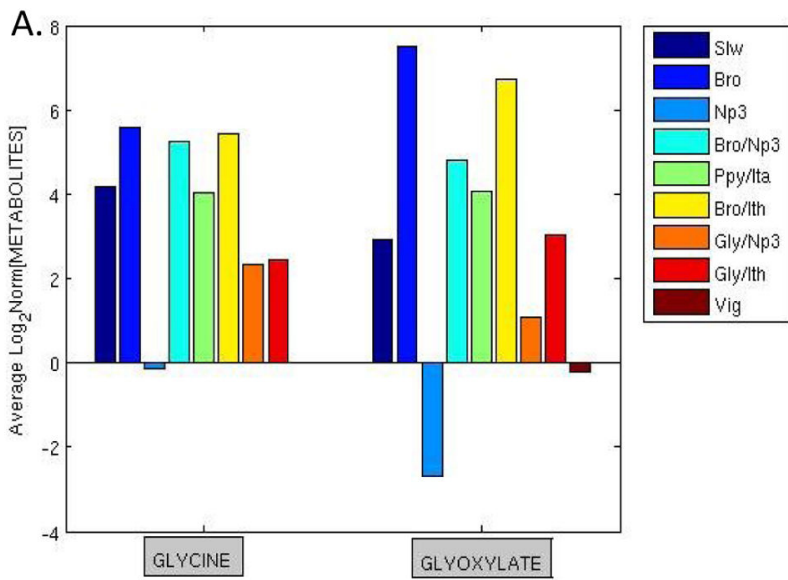

C.

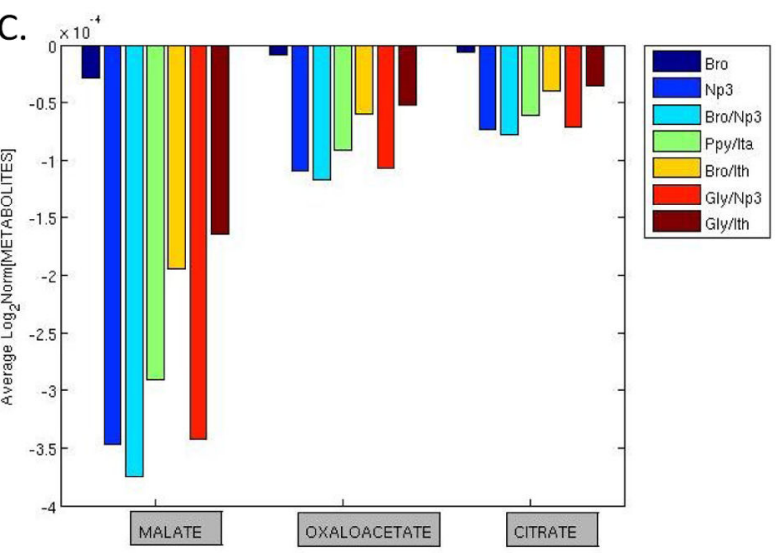

E.

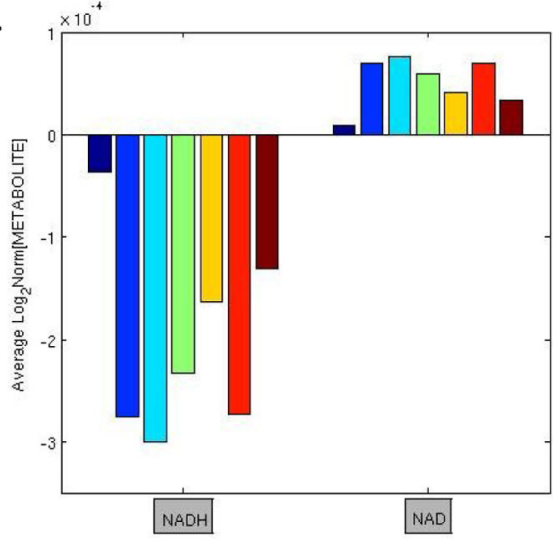

B.
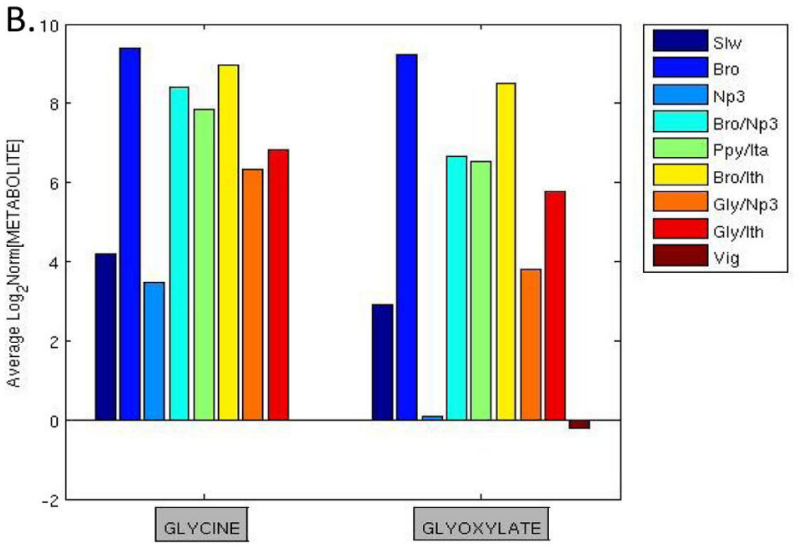

D.

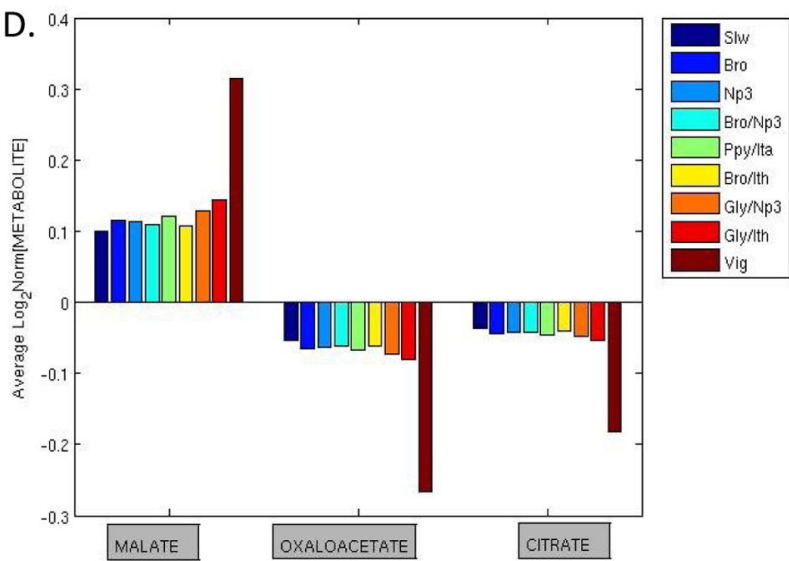

F.

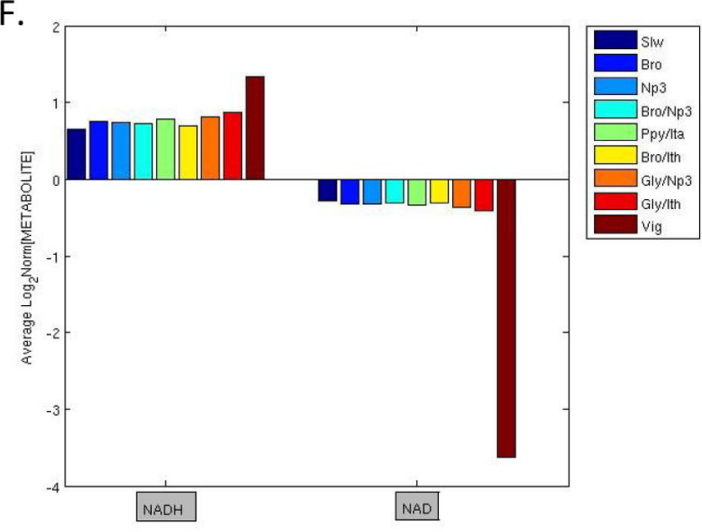

Figure 10.

Results of combined MS and ICL inhibition at 100x impacts several metabolites during simulated growth of Mtb under well aerated (A, C, E) and slow $\mathrm{O} 2$ depletion (B, D, F) conditions. Changes in the average metabolite concentrations normalized against the well-aerated, non-inhibited control are evaluated in comparison to the non-replicating persistence model (Slw) and the rapid oxygen depletion model where Mtb does not successfully replicate. 

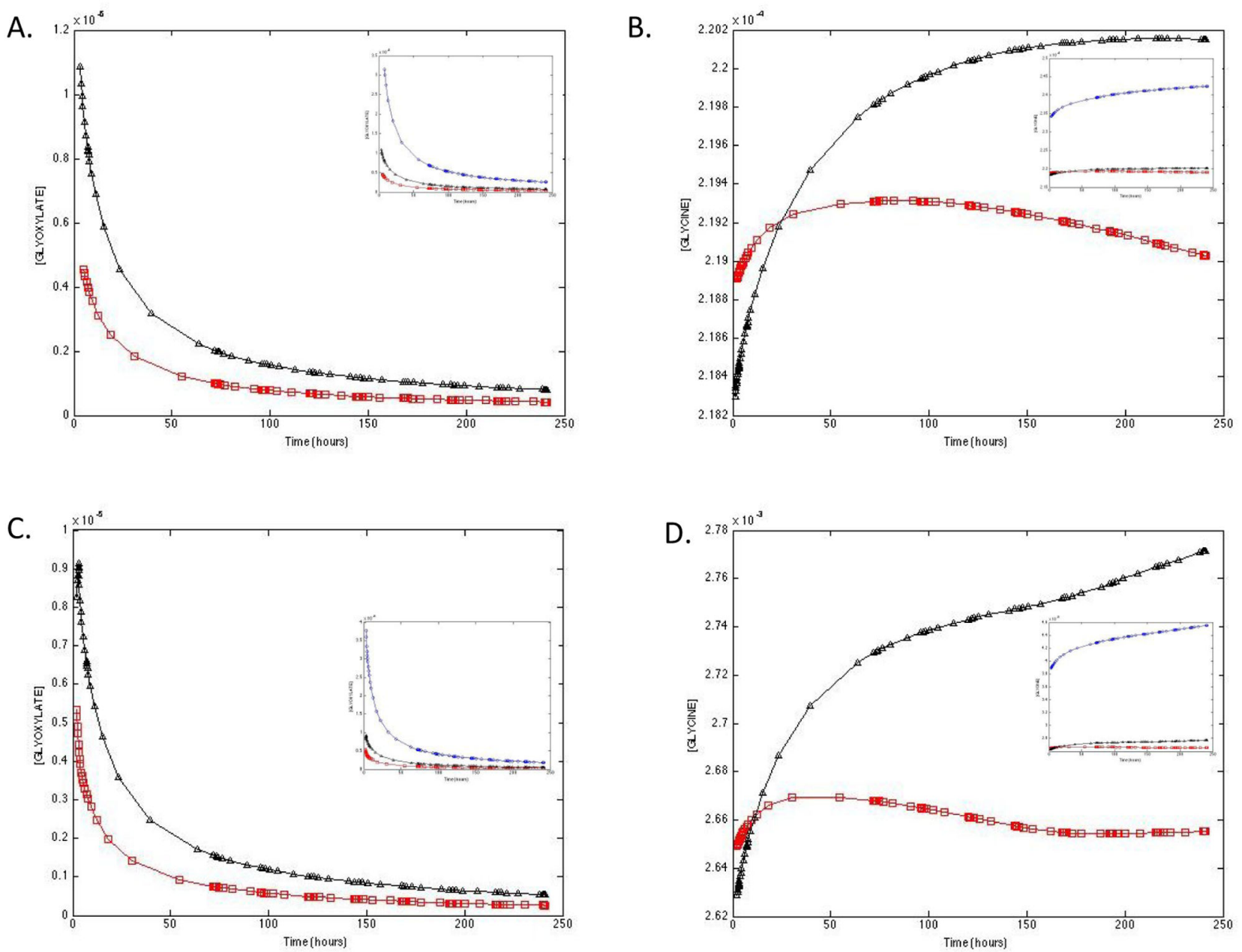

Figure 11.

Simulation of Mtb response and adaptation to inhibition by Np3. Comparison of inhibited metabolite (square) with metabolite levels when we account for Mtb genetic response (-triangle); inset figures compare results to non-inhibited model (-o-) for the aerated (A-B) and NRP (C-D) systems. When we account for Mtb adaptive response we observe higher levels of glyoxylate $(A, C)$ and glycine $(B, D)$, than in our initial inhibition models. 
Table 1

Reactions included in the model of TCA, glyoxylate, and GtG shunt.

\begin{tabular}{|l|l|}
\hline Enzyme Name & Reaction \\
\hline citrate synthase (CS) & $1 \mathrm{OA}+1$ ACCOA = 1 CIT + 1 COA \\
\hline aconitase (ACN) & $1 \mathrm{CIT}=1 \mathrm{ICIT}$ \\
\hline isocitrate dehydrogenase 1 (ICD1) & $1 \mathrm{ICIT}=1 \mathrm{AKG}$ \\
\hline isocitrate dehydrogenase 2 (ICD2) & $1 \mathrm{ICIT}=1 \mathrm{AKG}$ \\
\hline alpha-ketoglutarate decarboxylase (KGD) & $1 \mathrm{AKG}=1 \mathrm{SUCCSAL}$ \\
\hline succinic semialdehyde dehydrogenase (SSADH) & $1 \mathrm{SUCCSAL}=1 \mathrm{SUCC}$ \\
\hline succinate dehydrogenase (SDH) & $1 \mathrm{SUCC}+1 \mathrm{FAD}=1 \mathrm{FUM}+1 \mathrm{FADH} 2$ \\
\hline fumarase (FUM) & $1 \mathrm{FUM}=1 \mathrm{MAL}$ \\
\hline Malate dehydrogenase (MDH) & $1 \mathrm{MAL}+1 \mathrm{NAD}=1 \mathrm{OA}+1 \mathrm{NADH}$ \\
\hline isocitrate lyase 1 (ICL1) & $1 \mathrm{ICIT}=1 \mathrm{GLX}+1 \mathrm{SUCC}$ \\
\hline isocitrate lyase 2 (ICL2) & $1 \mathrm{ICIT}=1 \mathrm{GLX}+1 \mathrm{SUCC}$ \\
\hline Malate synthase (MS) & $1 \mathrm{GLX}+1 \mathrm{ACCOA}=1 \mathrm{MAL}+1 \mathrm{COA}$ \\
\hline glycine dehydrogenase (GDH) & $1 \mathrm{GLX}+1 \mathrm{NADH}=1 \mathrm{GLY}+1 \mathrm{NAD}$ \\
\hline
\end{tabular}




\section{Table 2}

Inhibitors of malate synthase.

\begin{tabular}{|c|c|}
\hline Compound & $\mathbf{K}_{\mathbf{I}}(\mathbf{u M})^{\boldsymbol{a}}$ \\
\hline Bromopyruvate & 60 \\
\hline Phosphoenol-pyruvate & 200 \\
\hline Oxalate & 400 \\
\hline Glycolate & 900 \\
\hline
\end{tabular}

${ }^{a}$ Data from [Smith, C. V. et al. 2003] 


\section{Table 3}

Inhibitors of isocitrate lyase.

\begin{tabular}{|c|c|c|}
\hline Compound & $\mathbf{K}_{\mathbf{I}} \mathbf{I C L 1}(\mathbf{u M})^{\boldsymbol{a}}$ & $\mathbf{K}_{\mathbf{I}} \mathbf{I C L 2}(\mathbf{u M})$ \\
\hline Bromopyruvate & 120 & 710 \\
\hline 3-nitropropionate & 3 & 110 \\
\hline Itaconate & 120 & 220 \\
\hline Itaconic anhydride & 190 & 480 \\
\hline
\end{tabular}

${ }^{a}$ Data from [Bentrup, K., et al. 1999; Scheer, M., et al. 2011] 